\title{
Salven nuestor palenta: análisis prospectivo de 150 niños, niñas y adolescentes de Chile sobre la educación ambiental del futuro
}

\author{
Save our palnet: prospective analysis with 150 children, girls,
} and adolescents from Chile about on the environmental education of the future

\author{
Gabriel Prosser Bravo ${ }^{a}$, Sonia Pérez Tello ${ }^{b}$, Marcela Pérez-Lienqueo ${ }^{c}$, \\ Carlos Prosser González ${ }^{d}$, Soledad Salazar-Sepúlveda \\ ${ }^{a}$ Escuela de Psicología, Universidad Academia de Humanismo Cristiano, Chile. \\ gabriel.prosser@uacademia.cl \\ ${ }^{b}$ Departamento de Psicología, Facultad de Ciencias Sociales de la Universidad de Chile. \\ sonperez@u.uchile.cl \\ ${ }^{c}$ Coordinadora del Programa de Innovación Pedagógica y Educación para la Sustentabilidad de Los Ángeles, \\ Chile.mperez@educacionlosangeles.cl \\ ${ }^{d}$ Consultor ambiental del Programa de Innovación Pedagógica y Educación para la Sustentabilidad de Los Ángeles, \\ Chile.prossercarlos@gmail.com \\ ${ }^{e}$ Licenciada en Psicología, Universidad de Chile. sole5013@gmail.com
}

\begin{abstract}
RESUMEN
Dada la actual crisis climática existe una tensión entre visiones esperanzadoras, catastrofistas y negacionistas del futuro. Los campos educativo ambientales han promovido visiones constructivas del futuro en las cuales, producto del accionar colectivo, se logran mitigar los efectos del cambio climático y adaptar a las comunidades a las nuevas condiciones de subsistencia. El presente estudio cualitativo reporta un análisis prospectivo de la educación ambiental, en base a cartas de 150 escolares que participan de un programa local chileno. Al respecto señalan un pool de medidas: acciones de gestión sustentable; de cuidado y preservación del medio ambiente; de comunicación y organización social; y de (auto)educación. A su vez, distinguen ciertas problemáticas glocales, como también actores y lugares claves en la construcción del mañana. Se discute la importancia de tomar esta sugerencias en términos políticos y de ofrecer más y mejores espacios para que prefiguren el futuro ambiental que desean vivir.

Palabras claves: educación ambiental, participación de niños, niñas y adolescentes, prospectiva ambiental, saber ambiental.
\end{abstract}

\begin{abstract}
Given the current climate crisis, there is a tension between hopeful, catastrophic and denialist visions of the future. Environmental education fields have promoted constructive visions of the future in which, as a result of collective action, the effects of climate change are mitigated, and communities adapt to new subsistence conditions. This qualitative study reports a prospective analysis of environmental education, based on letters from 150 schoolchildren who participate in a local Chilean program. In this regard, they point out a pool of measures: sustainable management actions; care and preservation of the environment; communication and social organization; and (self)education. They also distinguish certain glocal problems, actors and places that must be considered in the construction of tomorrow. Is discussed the importance of taking these suggestions in political terms and of offering more and better spaces to prefigure the environmental future they want to live.
\end{abstract}

Key words: environmental education, child and adolescent participation, environmental prospective, environmental knowledge. 


\section{INTRODUCCIÓN}

La situación ambiental ha generado un sinfín de predicciones ligadas a futuros catastróficos inevitables, de no mediar un cambio en la forma de relacionarnos con el medioambiente (World Economic Forum, 2019). Las predicciones científicas apuntan que para el 2100 serán $5^{\circ} \mathrm{C}$ promedio el aumento de la temperatura de nuestro planeta, lo que señala la urgencia de mejores respuestas ante la situación, como también la necesidad de consenso en torno al futuro que nos depara (Intergovernmental Panel on Climate Change, 2018).

Pese a que existe la posibilidad de que suba el nivel del mar, que se pierdan los arrecifes de coral, que sucedan grandes sequías, inundaciones, migraciones, desplazamientos y un aumento de los desastres naturales, se reconoce que las reacciones hasta ahora han sido insuficientes e incluso negacionistas, siendo el pasar a la acción un desafío para diversos entes (Organizaciones de Naciones Unidas, 2019). En este contexto, el eslogan de la Conferencia de las Partes (COP) número 25, de la Convención Marco de las Naciones Unidas sobre el Cambio Climático (CMNUCC) señala que el tiempo de actuar es ahora y que la acción climática depende de todos, resaltando la importancia de hacer en el presente y de que participen diversos/as sujetos para el logro de los objetivos propuestos.

Este tipo de discursos, junto con otros expresadas por activistas, académicos, movimientos sociales, populares e indígenas apuntan a que existe la posibilidad de encontrar una solución a la actual emergencia climática. En este sentido, junto con la idea de un futuro catastrófico inevitable, coexiste la de un futuro transformado o transformable, en el cual producto de la acción humana se logra mitigar efectivamente los impactos del cambio climático y adaptar a las comunidades de la mejor manera a las nuevas condiciones de existencia (González-Gaudiano, 2012; González-Gaudiano \& Meira-Cartea, 2020).

Ante esta situación, algunos actores/actrices sociales han denunciado no tener igual participación a la hora de plantear las soluciones futuras y construir los problemas tanto locales como globales. En especial, las personas menores de edad o consideradas jóvenes por la sociedad parecen despojados de su propio futuro y hábitat, a pesar de que una de las cosas que está en juego es su subsistencia (Hart, 2013; Mackey, 2012). La joven activista sueca Greta Thunberg ilustra esto en su discurso realizado el 13 de diciembre de 2018, en la COP 24, Katowice, Polonia:

En el año 2078 celebraré mi cumpleaños número 75. Si tengo hijos, tal vez pasen ese día conmigo. Tal vez me pregunten por ustedes. Tal vez pregunten por qué ustedes no hicieron nada cuando aún quedaba tiempo para actuar. Ustedes dicen que aman a sus hijos por encima de todo lo demás, y sin embargo les están robando su futuro frente a sus propios ojos

(Greta Ernman Thunberg, 13 de diciembre de 2018).

Este robo del futuro provendría de una postura de diversos sectores que promueven la inacción por medio del negacionismo, la catástrofe y la desesperanza, siendo relegado el rol de las personas menores de edad y de los jóvenes en las decisiones del cuándo, quiénes y cómo actuar frente al cambio climático (Liebel \& Gaitán, 2019). En respuesta a este escenario, la Educación Ambiental (EA) y la Educación para el Cambio Climático (EpCC) han sido campos disciplinares en los cuales se ha dado un espacio central a la niñez y la juventud en sus programas y acciones (García-Pérez \& Moreno-Fernández, 2015; Sauvé, 2013). Así, en 
las últimas tres décadas se elaboraron una serie de planes internacionales de EA enfocados en menores de edad, aunque sin el éxito esperado, no pudiendo generar un cambio global de comportamientos o conductas (O'Flaherty \& Liddy, 2017).

En este campo, se asume generalmente una postura a favor de la participación infantojuvenil en las temáticas ambientales, climáticas y educativas (Tréllez, 2015). Existen sin embargo experiencias donde la participación de niños, niñas y adolescentes (NNA) es apenas simbólica o superficial (Pramling-Samuelsson, 2011), dificultándose con esto la posibilidad de los más jóvenes de indagar sobre las problemáticas de su territorio y de actuar en pos de construir una sociedad más sustentable y justa (Gadotti, 2008). Por ilustrar esto, en un estudio empírico con docentes de EA realizado por Matos, Pasek, Peña y Briceño (2018), se evidenció que solo el 17\% de los/as profesores incluidos en la muestra promovía actividades realmente participativas.

Dicho esto, el presente artículo busca indagar en dos cruces: por un lado, respecto del futuro, existiendo posiciones catastróficas y negacionistas, como también otras activas y con esperanza en la construcción de un futuro mejor; y por otro, una educación participativa versus otra de corte transferencial que relega a NNA a la posición de meros receptores. Es, en palabras de Edgar González Gaudiano y de Pablo Meira Cartea (2020), el choque entre las educaciones sobre el clima y las educaciones para el cambio.

Las primeras están marcadas por la apatía a la emergencia y las vulnerabilidades climáticas, por lógicas comunicacionales de transferencia que invisibilizan las causas de la crisis, y configuran el problema del cambio climático como algo demasiado inmenso, respecto de lo cual la humanidad poco puede hacer tanto individual como colectivamente. Las segundas, por su parte, promueven transformaciones en diversos sistemas de la vida humana en pos de mejorar nuestra eficacia colectiva a la hora de enfrentar el cambio climático. Una EA o EpCC que, como proponen los autores, se encargue rápidamente de poner en conciencia del estado de colapso en el cual nos encontramos, para desde ahí, construir nuevas posibilidades y acciones (González-Gaudiano \& Meira-Cartea, 2020). En palabras de la educadora ambiental peruana Eloisa Tréllez (2002):

La educación ambiental debe rebelarse también a ser reducida a un conjunto de prácticas de naturaleza, a centrarse exclusivamente en la contaminación y en los futuros nefastos que nos esperan, a convertirse en la última voz que clama antes del desastre. El rol de los procesos educativos ambientales crece día a día en la medida en que somos más conscientes de la necesidad, no solo de remediar los problemas ambientales con parches cada vez más pequeños e inútiles, sino de aportar en la construcción de un futuro diferente, con miras a la sustentabilidad, a la equidad, a la valoración de lo diverso y lo múltiple, a la paz y el equilibrio (Tréllez, 2002, p. 8).

Por tanto, la presente investigación problematiza la potencialidad de la participación infanto-adolescente en la EA/EpCC y la necesidad de incluir a NNA en ejercicios que nos permitan prepararnos y anticiparnos a nuestro futuro ambiental. En este sentido, el mañana ha estado presente solo en algunas de las preocupaciones de la EA y de los proyectos ambientales, relegando en ocasiones los imaginarios colectivos a construcciones imperantes y desesperanzadoras en torno a la catástrofe climática (Hicks, 2006; Strife, 2012; Tréllez, 2015).

Este texto parte de estas consideraciones para analizar mediante un estudio de caso como los NNA pueden, una vez vividos procesos educativos ambientales o de cambio 
climático, convertirse en actores y actrices con capacidad de visibilizar y construir el futuro que desean y creen posible ante la actual crisis. Para dar contexto a esto, a continuación revisaremos las diversas líneas de estudio que vinculan el futuro con la EA, teniendo en consideración la importancia de la participación activa de NNA en este campo.

\subsection{EL FUTURO Y LA EDUCACIÓN AMBIENTAL}

Articular el futuro ambiental y la participación de NNA en la EA tiene como objetivo garantizar los DDHH de estos, mejorar los programas e intervenciones educativo ambientales y develar el fuerte impacto que tiene en la acción pro ambiental infanto-adolescente, tanto la visión de futuro de los NNA, como el grado de participación que se les permite en las experiencias educativas. Según estudios recientes, la EA logra mejores resultados si es que promueve una mayor participación del estudiantado (Cincera, Boeve-de Pauw, Goldman \& Simonova, 2019) y si es que transmite una visión del futuro transformable y constructiva (Kerret, Orkibi \& Ronen, 2014). Aún más si es que producto de los procesos educativos se les permite vislumbrar, construir y transformar activamente su futuro (Schröder, Wals \& van Kopper, 2020).

De aquí han surgido dos interesantes líneas de estudio de la EA: 1) una línea interesada por estudiar y/o medir de qué manera impacta la EA en las visiones de futuro, y que consecuencias tienen esas visiones en la conducta pro ambiental (Ojala, 2012a; 2012b; Strife, 2012); y 2) una serie de investigaciones que buscan indagar cómo la educación puede abrir caminos y experiencias participativas para que los/as educandos imaginen y construyan materialmente el futuro que desean y creen posible (Hicks, 2014; Malone, 2013; Tsevrini \& Panayotatos, 2011).

Dentro del primer grupo de estudios existe la hipótesis de que ante experiencias educativas ambientales los educandos desarrollan cierto grado de optimismo o esperanza respecto del futuro climático, lo que más tarde decanta en un mayor bienestar y comportamiento pro ambiental (Kerret, Orkibi \& Ronen, 2014). Al respecto, Strife (2012) realizó un estudio con 50 NNA de entre 10 y 12 años de Estados Unidos sobre el futuro ambiental del planeta. El análisis de dibujos sobre cómo sería el planeta en 100 años arrojó que la mayoría de estos NNA experimenta sentimientos pesimistas y apocalípticos respecto del futuro, lo que promueve una fuerte inacción en ellos. Contrario a esto, solo algunos NNA mostraron una visión positiva, dibujando energías renovables, ciudades sustentables y amigables. Mientras los primeros decían educarse de temas ambientales principalmente por televisión y películas, los segundos mostraron un grado de conocimiento de la temática mucho mayor debido una multiplicidad de fuentes de conocimientos (e.g. familia, escuela, medios de comunicación).

En otro estudio con 624 adolescentes de Suecia, Ojala (2015) identificó dos tipos de esperanzas de futuro vinculadas a experiencias educativas: por un lado, una esperanza constructiva que fomenta el compromiso y la participación activa del estudiantado, producto de que creen que existen soluciones para enfrentar el futuro climático; y por otro, una esperanza basada en la negación, en la naturalidad del problema o en la confianza de que la evolución tecnológica nos salvará, lo que justifica el no comprometerse al respecto. Tal como en otros estudios de la autora (Ojala, 2012a y 2012b), este permitió constatar que la EA tiende a desarrollar una esperanza constructiva basada en conocimientos ambientales certeros, aunque no siempre traduciéndose en comportamientos o conductas (Ojala, 2012a). 
El segundo grupo de trabajos, se enmarca en la tradición de los Future Studies, y en la EA tiene en David Hicks (2014) su mayor representante. Según el autor, la importancia de este tipo de estudios radica tanto en detectar los principales imaginarios e imágenes que existen sobre el futuro, como también en ayudar a los colectivos humanos a ser activos en la construcción del futuro que desean (Hicks, 2006). Mediante diversas técnicas se espera ayudar a visualizar un futuro más plausible para las personas, de manera que sepan diseñar una hoja de ruta para llegar a este (Hicks, 1996). Se trata de dar luz al presente mediante esclarecer las pautas que nos permitan generar los cambios que creen posibles para el futuro.

Uno de los estudios fundacionales fue realizado por el mismo Hicks (1996) con 90 estudiantes universitarios ingleses, a los cuales les pregunto por las características del futuro que deseaban vivir para el año 2020, siendo destacado por sobre todo un futuro sustentable, verde y con relaciones sociales amigables. 35 años después sabemos que estos imaginarios estuvieron lejos de cumplirse (ONU, 2019) y que, como indican los estudios de la primera línea aquí mencionada, esta dificultad por construir tal futuro se debe en parte a una visión desesperanzada o negadora de este (Hicks, 2014; Ojala, 2015).

Dentro de esta segunda línea también cabría mencionar la experiencia levantada por Tsevreni \& Panayotatos (2011) con 59 NNA de entre 9 a 12 años de dos escuelas de Grecia. El estudio permitió constatar que los NNA no se involucraban en actividades ambientales o no opinaban mayormente en estas debido a que consideraban que no serían escuchados. A partir del proyecto se les pidió que elaborasen propuestas para el futuro de su barrio, teniendo como orientación mejorar su calidad de vida, lo que trajo por consecuencia un aumento en su comportamiento ambiental, un mayor compromiso con el tema, una mejora en su autoestima y una serie de propuestas elaboradas mediante maquetas, collages y otros para expresar cómo querían fuesen sus vecindarios (Tsevreni \& Panayotatos, 2011).

Otro estudio relevante al respecto fue realizado por Malone (2013) con 150 NNA australianos de entre 5 a 10 años. En este, se reporta una investigación-acción participativa que tuvo por objetivo que estos niños y niñas pudieran evaluar que tan amigables y ecológicos eran su barrios, para después visionar que cambios harían a futuros en estos con el fin de mejorarlos. Producto de los talleres realizados fue posible: i) elaborar vías de transito que mejoraron la conectividad entre sus lugares significativos y los espacios naturales, ii) crear caminos peatonales en los parques con iconografía diseñada por los NNA, iii) pintar con arte de la tradición aborigen de la zona murales y iv) separar las ciclovías de las pistas de autos.

En este marco global, son pocos los estudios de casos realizados en Latinoamérica y reportados en español, siendo difícil aseverar si existen diferencias o no respecto de otros futuros deseados y creídos posibles por NNA del mundo. Así mismo, algunos ejercicios de futuro se han focalizado en imágenes globales como "el futuro del planeta" o en abordajes locales como "el futuro de tu barrio", perdiendo en ocasiones la posibilidad de analizar la glocalidad del futuro que nos depara. Más aún, es difícil rastrear antecedentes académicos en los cuales se les haya preguntado de manera directa a los NNA respecto de cómo construirían la EA o la EpCC del futuro.

Por esto, el objetivo del presente estudio fue identificar el futuro que desean y creen posible para la EA un grupo de NNA involucrados en un programa local de EA realizado al sur de Chile. En base a la línea de participación y de estudios del futuro de la que se ha venido hablando esto involucra preguntarles por el qué hacer ante la crisis climática, 
profundizando más allá del qué creen irá a pasar. En este sentido, un objetivo específico del estudio es describir que acciones o iniciativas se les ocurren a los NNA a la hora de prefigurar la EA y nuestra respuesta climática ante el futuro.

\section{MATERIALES Y MÉTODO}

Se siguió la tradición cualitativa de la investigación, la cual sostiene que es posible entender la realidad social mediante la interpretación de las interacciones que en esta se dan (Seamon \& Gill, 2016). A su vez, Íñiguez y Antaki (1994) sostienen que tal realidad social es posible de ser estudiada mediante el análisis de las narrativas de los sujetos que en ella conviven.

En particular, el presente estudio ve el futuro catastrófico e inevitable de la crisis climática como aquella imagen instituida y como una representación social imperante de lo que depara el futuro (González-Gaudiano, 2012; Tréllez, 2015). En torno a esta imagen existe la posibilidad de habilitar campos y experiencias en donde poner en cuestionamiento los efectos de tal visión, y así abrir espacios para construir, junto con sujetos y comunidades, "un futuro provisto de toda utopía" (Hicks, 2014; Leff, 2011, p. 2).

Pensar los futuros otros mediante análisis prospectivos, permite orientar la acción humana hacia la construcción de futuros alternativos, y no necesariamente al análisis de tendencias, predicciones o vaticinios (Hicks, 1996). Supone identificar los deseos, intereses e imaginarios de los individuos y colectivos, en pos de actuar de manera tal que permita acercarnos a tales futuros deseados. En el caso de la EA, estas metodologías buscan incorporar la voz y opinión de sus participantes, mediante conocer e integrar sus visiones del futuro (Hicks, 2006). El potencial que ve el campo en estos ejercicios prospectivos radica en que:

En general, el futuro se toma como un espacio neutral, sobre el cual es posible construir. Ello genera rutas de consenso y permite recrear de manera conjunta los escenarios de aproximación al futuro considerado posible y deseable (futurable) para la comunidad (Tréllez, 2002, p. 14).

\subsection{CASO DE ESTUDIO: 150 CARTAS DE NIÑOS, NIÑAS Y ADOLESCENTES DEL PROGRAMA DE INNOVACIÓN PEDAGÓGICA Y EDUCACIÓN PARA LA SUSTENTABILIDAD (PIPES)}

El PIPES es diseñado el año 2014 por la Dirección de Administración de Educación Municipal (DAEM), de la Municipalidad de Los Ángeles, Chile. Según el Plan Anual de Desarrollo de la Educación Municipal, tiene por objetivo integrar en las instituciones y en la cultura educativa de la comuna, ciertos conocimientos y capacidades relacionadas con el cuidado y protección del medioambiente, como también con la mitigación y adaptación al cambio climático, siempre teniendo como norte la preservación de la riqueza ecológica de la zona (Alonso, 29 de septiembre de 2018).

Se realiza en más de 51 establecimientos, alcanzando un total aproximado de 12.500 estudiantes (la mitad de la matricula municipal) y se estima que llega a una población indirecta de 65.000 personas (1/3 de la población comunal). Es implementado por un equipo interdisciplinar compuesto por dos profesionales municipales y una serie de docentes que coordinan la implementación en las instituciones educativas. En conjunto forman la Red 
de Comunidades Educativas Sustentables, la que vela por el cumplimiento de los objetivos del programa.

En él, los niños, niñas, adolescentes y jóvenes cuentan con diversas acciones de las cuales pueden ser parte. En primer lugar, en cada establecimiento existe un taller ambiental permanente donde se trabaja con estudiantes en la temática y se realizan acciones de difusión, reciclaje, cuidado de luz y agua, y limpieza de los espacios. A su vez, esta instancia ha generado unidades de producción vegetal, se han plantado árboles nativos y se han construido puntos limpios, viveros, invernaderos, etc.

Saliendo del taller, pueden participar de actividades de vida saludable, del Club de Forjadores Ambientales y de la celebración del Día de la Tierra, del Agua y del We Tripantu, o año nuevo mapuche. Fuera de la escuela, participan de actividades de educación al aire libre y en eventos comunales como el Carnaval del Reciclaje y la Feria de Educación para la Sustentabilidad. En estos, los NNA visibilizan el trabajo anual que realizan en su establecimiento.

\subsection{PARTICIPANTES}

En el año 2018, la DAEM decide realizar una evaluación municipal multinivel del PIPES, desarrollando un taller de actividades para evaluar y proyectar, junto con escolares, la implementación del programa en sus escuelas, en la comuna y en el país. A esto accedieron de manera voluntaria seis directivos de escuelas y liceos municipales, siendo tres provenientes de establecimientos rurales y tres de urbanos.

Dada la heterogeneidad de los tamaños de curso, se trabajó con aquellos NNA que participan del taller ambiental o de las actividades ambientales. Por este motivo, se dio una distribución heterogénea de la cantidad de estudiantes por escuela partícipes de la evaluación (Ver Tabla 1). En total, participaron 150 niños, niñas y adolescentes de entre 6 a 14 años, siendo mujeres el 53,37\% de estos.

Tabla 1. Participantes

\begin{tabular}{|l|l|c|c|c|l|}
\hline NE & Establecimiento & $\mathrm{N}$ & Mujeres & $\underline{x}$ por curso & Ruralidad \\
\hline E1 & Liceo Santa Fe & 17 & $47,05 \%$ & 24 & Rural \\
\hline E2 & Escuela República de Alemania & 23 & $47,61 \%$ & 30 & Urbana \\
\hline E3 & Escuela la Quebrada & 26 & $58,33 \%$ & 8 & Rural \\
\hline E4 & Escuela Nieves Vásquez Palacios & 22 & $54,54 \%$ & 25 & Urbana \\
\hline E5 & Escuela Thomas Jefferson & 32 & $59,37 \%$ & 30 & Urbana \\
\hline E6 & Escuela el Nogal & 30 & $53,33 \%$ & 20 & Rural \\
\hline
\end{tabular}

Fuente. Elaboración propia. NE: Número de escuela en los resultados. 


\subsection{TÉCNICAS DE PRODUCCIÓN DE LA INFORMACIÓN}

Se reiteró el taller en las seis inmediaciones, adecuándolo a la cantidad de integrantes, el espacio y los recursos disponibles. Este se dividió en tres fases: en una primera, tuvieron que detectar de manera individual y colectiva aquellos aspectos positivos y negativos del desarrollo del PIPES en su escuela; en una segunda, jugaron una versión del piedra, papel y tijera adaptado a la cadena trófica de la Zona Sur de Chile y, tercero, escribieron una carta bajo la instrucción "qué le dirías a un/a extranjero/a sobre cómo desarrollar en el futuro la EA y los proyectos ambientales de su país"1. De esta última nace este trabajo.

El taller integró las recomendaciones de Trelléz (2002) y Hicks (2006) para hacer análisis prospectivo, puesto que en una primera fase fue necesario realizar un autodiagnóstico de las problemáticas locales y escolares, para después construir una visión crítica sobre la EA en su escuela. Es este primer paso en tiempo presente, el que facilita el que después los NNA sean capaces de pensar en clave presente-futuro y de proyectar la EA a otras latitudes.

Por esto, se realizó un ejercicio similar al visioning 2 (visionado), que según Fernández Guell (2011) permite abordar temas complejos e inciertos y resulta adecuado para cuando no existe una gran masa de datos precedentes, e inclusive para aquellas situaciones históricas, como la actual crisis climática, donde los cambios son tan acelerados y relevantes que requieran de una actualización constante. Este tipo de estudio de futuro es utilizado en investigaciones de EA para obtener claridad sobre las visiones de futuro existentes y como llegar a prefigurar los deseos comunes mediante acciones colectivas (Hicks, 2006).

\subsection{TÉCNICAS DE ANÁLISIS DE LA INFORMACIÓN}

Se realizó un análisis prospectivo de tipo exploratorio y anticipatorio (Rodríguez, 2014) ${ }^{3}$, identificándose de manera exploratoria los tópicos e imaginarios que son mencionados por los y las escolares, con el fin de hacerse una idea de aquello que más desean e imaginan para el futuro. Posteriormente, estos deseos fueron analizados considerándoles como potenciales políticas, programas o acciones ambientales que pudieran realizarse, poniendo atención a la detección de nudos críticos, oportunidades y problemáticas.

Para el tratamiento de la información se transcribió y escaneó cada una de las cartas, las que fueron de una o dos planas de extensión. Estas, fueron divididas según escuelas y se asoció un $\mathrm{N}^{\circ}$ a cada estudiante, sin distinguir el género de este/a. Posteriormente, se trabajó el material escrito mediante un análisis de contenido cualitativo (Seamon \& Gill, 2016) el que permitió una dimensión de ciertos tópicos mencionados. Una vez formulado un pool inicial, se procedió a una relectura cruzada de las cartas por parte de los/as investigadores, buscando categorías definitivas que fueran representativas.

En ocasiones, cuando tenían dudas respecto de qué escribir, se les dio indicaciones aclaratorias del tipo "imagina que en su país el desarrollo de la EA es aún temprano", "imagina que las escuelas quieren ser medioambientales", "qué crees que hace medioambientalmente buena a tu escuela y que otros/as deberían imitar", "qué proyectos ambientales deberíamos hacer en Chile y en otros países".

2 Técnica utilizada en contextos organizacionales para proyectar el futuro desarrollo de una empresa, red o proyecto; o para iniciar un nuevo proceso de diseño, teniendo como criterios la sustentabilidad, el trabajo colectivo, la idea de proceso y la definición de acciones envolventes que son necesarias para poder llevar a cabo tales objetivos de desarrollo (Fernández-Guell, 2011).

3 Este tipo de análisis se utiliza en política pública como un insumo para las fases de evaluación y de planificación, puesto que permite anticiparse a problemáticas, identificar intereses y adquirir conciencia de los imaginarios de la población (Tréllez, 2002; 2015). 
Finalmente, para la elaboración de los resultados se procedió a hilar una narrativa que reflejara el análisis, profundizando en aquellos elementos más relevantes en los imaginarios y visiones de los/as participantes. Producto de esto, fue posible identificar las sugerencias y potenciales acciones proambientales en las subcategorías que sirven de títulos para los resultados.

\subsection{CONSIDERACIONES ÉTICAS DE LA INVESTIGACIÓN}

Usando los niveles de participación de Roger Hart (2013), el taller alcanzó el nivel consultados e informados, puesto que los NNA comprendieron el objetivo del proyecto, entendieron que su rol estaba diseñado por adultos/as y accedieron voluntariamente a hacer de asesores/as. A su vez, desde Trilla y Novella (2001), se empleó una participación proyectiva, haciendo uso de sus impresiones y visiones para proyectar e imaginar la EA y los proyectos ambientales que se debieran forjar en el futuro.

La investigación contó con la aprobación y colaboración de autoridades locales y se les solicitó el consentimiento verbal a los/as participantes. Se siguieron principios éticos de la investigación con NNA, tales como adecuar las actividades al rango etario, propiciar dinámicas que faciliten la voz de todos/as, abrir espacios de consulta previos y posteriores al taller, garantizar el anonimato y confidencialidad, hacer explícita la posibilidad de no participación y retiro, explicar los motivos por los cuales se considera que sus opiniones eran fundamentales para la investigación, como otras (Navas, Martínez, Valdebenito \& Castillo, 2018).

\section{RESULTADOS}

Los presentes resultados son síntesis de lo que, en palabra de los y las NNA, son "los pasos para cuidar tu colegio, ciudad, país" (Estudiante 6, E1). Este proceso, estaría asociado tanto a "mejorar las escuelas" (Estudiante 17, E5) como a explicar las acciones necesarias para "convertir una escuela común en una escuela medioambiental" (Estudiante 7, E5). Pese a que el desafío de pasar de lo común a lo medioambiental es complejo y excede tanto a la educación como a la escuela, los/as escolares señalan que "hay muchas maneras de hacerlo" (Estudiante 3, E5), y ofrecen "consejos", "tips", "sugerencias" o "ideas" que contribuirían a vivir el futuro ambiental que desean.

Por tanto, fue posible identificar una serie de recomendaciones tanto para mejorar la educación y la sociedad, como para volverlas más ambientales. A su vez, algunos dieron las "gracias por leer nuestras opiniones sobre el medio ambiente", señalando que estos ejercicios sirven "para que así en el futuro el planeta no esté tan contaminado como ahora y que los niños podamos aprender de esto y ser mejores personas" (Estudiante 4, E2).

Respecto del futuro que desean y aconsejan desarrollar fue posible identificar dos grandes categorías; en primer lugar, aparecen una serie de acciones ambientales fundamentales para el desarrollo de un futuro sustentable y equitativo. Aquí encontramos acciones calificadas como acciones de gestión, de cuidado y preservación, de comunicación y organización, y de (auto)formación; en segundo lugar, se identificaron nodos críticos de los cuales debemos hacernos cargo, destacando ciertas problemáticas, ciertos entes y lugares. 


\section{1. "EL TIEMPO DE ACTUAR ES AHORA": ACCIONES PROAMBIENTALES PARA AVANZAR HACIA EL FUTURO QUE DESEAMOS}

Esta categoría incluye acciones que los y las escolares creen fundamentales para lograr que una persona, escuela, ciudad o sociedad se vuelva más "medioambiental" o "sustentable". Haciendo uso de parte del eslogan de la COP 25, dado que el tiempo de actuar es ahora, debemos tener claro qué acciones son determinantes en la construcción del futuro que deseamos, pues esto nos permitirá guiar y priorizar las iniciativas, en pos de lograr este futuro.

Las cuatro subcategorías no responden a diferenciaciones hechas por estudiantes, sino a cómo se ha decidido agrupar, con fines analíticos, una serie de recomendaciones que coexisten a un mismo nivel discursivo. La primera, reúne a aquellas acciones de gestión ambiental sustentable, entendiendo que, pese a que todas las sugerencias son clasificables como este tipo de acción, las señaladas aquí refieren a los sistemas de gestión ambiental tradicionales, como lo son el sistema de residuos, energético, de producción vegetal, etc.

Aparecen acciones de cuidado y preservación, las que han sido distinguidas dada la frecuencia del verbo cuidar en las cartas, lo que habla de la importancia de esta acción para los/as NNA. También encontramos acciones de comunicación y organización, asociadas a cómo involucrar a más personas en las acciones ambientales, cómo difundimos las ya realizadas y qué necesitamos para entender las diferentes necesidades y formas de participación. Por último, se distinguieron acciones (auto)formativas, ubicando aquí instancias, maneras y actores claves para el proceso de enseñanza-aprendizaje.

\subsubsection{Acciones de gestión sustentable}

Aquí encontramos sugerencias relacionadas con la producción vegetal, la gestión de residuos y la infraestructura. Respecto de lo primero, señalan que para un futuro más sustentable es necesario "plantar más árboles y flores, porque hay niños y adultos que están cortando los árboles y los talan. Para tener más naturaleza y aire puro para los niños" (Estudiante 18, E2). De la cita se desprende que esta flora es necesaria para mitigar el actual impacto humano y para mejorar las condiciones de vida de las personas.

Dentro de la escuela los/as estudiantes señalan que se puede "hacer un vivero, regar las plantas, poner más plantas" (Estudiante 19, E1), destacando las diversas unidades de producción vegetal que pueden existir. También, sugieren "tener más puntos verdes, un depósito de sobras de frutas, de vegetales, para luego volverlo compost y alimentar a las plantas" (Estudiante 10, E1). Con el mismo fin de reutilizar los desechos orgánicos de la escuela, recomiendan "hacer una casa de lombrices" (Estudiante 9, E1).

Señalan también, que estas acciones de enverdecimiento pueden suceder fuera de los establecimientos educacionales, siendo posible, por ejemplo, "plantar más árboles en las comunas" (Estudiante 5, E2) o "poner más áreas verdes en las partes más céntricas. Plantar árboles en lugares públicos en donde se necesitan" (Estudiante 23, E2). En este sentido, la arborización, puede realizarse tanto en sectores considerados estratégicos como necesarios.

Relacionado con lo segundo, los y las estudiantes refieren a acciones de las " $R$ " de la gestión de los residuos domiciliares y escolares. Primero, reconocen la necesidad de "empezar a ocupar las 3R; reduce, reutiliza, recicla" (Estudiante 30, E5) y de "reinventar la basura" (Estudiante 6, E6). Para esto recomiendan "hacer actividades con los tetra pack, hacer lapiceros, bolsas de género" (Estudiante 15, E6) u “ocupar cosas viejas para hacer 
otras cosas, como vasos, hacerlos maceteros" (Estudiante 3, E6). Muchos/as estudiantes hablan del reciclaje como una acción fundamental que puede realizar cualquier persona con pocos recursos.

A nivel de establecimiento educacional un/a estudiante señala que:

para que sea una escuela ambiental necesitan enseñarles a reciclar, necesitan saber botar la basura en un lugar y ese lugar puede ser el punto limpio, pero el punto limpio tiene para las botellas, cartón, papel y latas y tienen que saber botar. En mi escuela todos los salones tienen un punto limpio, un lugar donde botar los papeles, necesitan contenedores (Estudiante 2, E5).

Otra/o destaca que deberían "poner afiches, basureros para reciclar la basura, diferentes basureros para reciclar" (Estudiante 29, E6). En ambas cartas podemos observar la importancia tanto de educar e informar, como de proveer de la infraestructura para que se puedan realizar acciones de reciclaje. Esto refuerza otra/o escolar cuando señala que hay que colocar "basureros que tengan logo de papel, lata, plástico, vidrio" (Estudiante 16, E2). Pero no solo los basureros son la infraestructura que imaginan los y las escolares, algunos/ as señalan que "sería bueno colocar energía eólica en los colegios" (Estudiante 14, E2), que se puede "hacer un acopio" (Estudiante 15, E1) o, como ya se mencionó, un vivero.

Los y las estudiantes mencionan que les gustaría que existan "más lugares verdes para que todas las personas jueguen libremente sin peligro" (Estudiante 8, E6) o "tener espacios verdes, decorar con cosas del medio, plantar muchas plantas, transformar cosas reciclables y poner juegos ecológicos" (Estudiante 25, E6). Como se ve, haciendo referencia a que tener más áreas verdes facilitará las posibilidades de ocio y esparcimiento de las personas. Otra infraestructura que recomiendan son los puntos limpios, pues facilitan que las comunidades aledañas almacenen y reciclen papel, cartón, botellas plásticas, vidrio y latas.

\subsubsection{Acciones de cuidado y preservación}

La preservación del medioambiente y el cuidado de los recursos forma parte de las acciones de gestión ambiental, pero ha sido diferenciada por la reiteración de la palabra cuidar para referir a acciones que para las y los NNA son importantes. Aquí, el cuidar es asociado a los seres vivos, la salud de las personas, a los recursos energéticos y la limpieza. Dentro de los seres vivos de mayor importancia destacan las plantas y los árboles. Un/a estudiante ejemplifica esto señalando que "cuando vean nuevas flores bonitas que no la tome y la coloca en su colegio [...]. Para mí es importante esto porque puedo cuidar el planeta y salvar las plantas" (Estudiante 9, E6).

Esta importancia que tiene la flora hace que se nieguen a acciones que las perjudican, señalando el "no maltratar los árboles. Tener un mejor cuidado con las plantas, cuidar las flores [...], tener escuelas y cuidar el jardín" (Estudiante 21, E6). Esto significa "mantener a los seres vivos, mantener las flores bien regadas (Estudiante 15, E1), "no talar árboles" (Estudiante 20, E2) o "no arrancar las plantas" (Estudiante 21, E2). En general, que se prohíba toda acción que dañe estos seres vivos y que se incentive toda aquella que tienda a preservarles.

Los y las escolares señalan que es importante cuidar a las plantas "para que el día de mañana no nos falten" (Estudiante 21, E3), puesto que "si cortas muchos árboles, el aire 
se podría acabar" (Estudiante 2, E4). Destacan la importancia también de "no dañar las plantas, porque las plantas puede que nos den alimentos" (Estudiante 13, E4) y que inclusive "cuidar las plantas" contribuye a "una mejor escuela, que sea llamativa" (Estudiante 25, E6). Así, el cuidar el mundo vegetal responde también a inquietudes sobre el futuro que tienen los y las NNA.

Otro ser vivo de gran importancia son aquellos pertenecientes al reino animal, siendo un señalamiento frecuente el que hay que "ayudar a los animales" (Estudiante 14, E6) y evitar que "maltraten a los animales, (que) no le peguen a los animales" (Estudiante 2, E2). En especial, hablan de "cuidar más a las mascotas" (Estudiante 5, E2) o animales domésticos: "deberían tratar de construir algunas casitas para los perritos. No importa que sean pequeñas, es solo para que se refugien, porque los perritos son seres vivos, igual que nosotros" (Estudiante 24, E3).

Respecto de la salud y el bienestar de los animales humanos, remarcaron que no hay que "fumar porque da cáncer" (Estudiante 3, E4) y que "si fumas intoxicas a tu bebe e intoxicas a tus pulmones" (Estudiante 4, E4). Señalan lo importante de regular el cigarro pues "puede provocar incendios" (Estudiante 13, E4). Respecto de nuestra salud, un/a escolar destaca el medioambiente, pues "es importante para nuestra existencia física y psicológica” (Estudiante 17, E1).

Los y las NNA mencionan reiterativamente el cuidar la luz y el agua, la primera mediante "apagar la luz cuando no la usas", y la segunda al "cerrar las llaves de agua cuando no la ocupas" (Estudiante 17, E6), al evitar "no dejar la llave dada" (Estudiante 8, E3) o "cortando el agua cuando te cepillas los dientes" (Estudiante 22, E2). Muchos/ as estudiantes le dan una importancia vital al tema del agua "porque nos sirve para beber" (Estudiante 17, E4). Como ya se mencionó, para los y las escolares es importante "mantener limpio" (Estudiante 30, E5), ya que mantener "limpias las salas" (Estudiante 3, E5) permite que los establecimientos educacionales puedan "ser lindos y limpios" (Estudiante 23, E6).

\subsubsection{Acciones de comunicación y organización}

Estas acciones tienen un carácter apelativo hacia otro/a, en pos de que este/a se sume a las iniciativas, lo que se refleja en el uso de las palabras "invitar", "motivar", "convencer". Es "tratar de incentivar a la misma escuela o a tus compañeros" (Estudiante 30, E5), "incitar a los niños" (Estudiante 3, E1) o "motivar a los demás a cuidar nuestra naturaleza" (Estudiante 23, E2). Sea cual sea la forma, se trata de involucrar a un/a otro/a en acciones proambientales. Según escolares, una medio para lograr esto es la difusión, como por ejemplo "poner afiches" (Estudiante 7, E6).

También destacan el liderazgo como algo importante para el involucramiento, al igual que "invitar a la comunidad" (Estudiante 30, E5), entendiendo a esta como algo más amplio que los/as docentes y estudiantes. Otros aspectos organizativos importantes son "trabajar en equipo" (Estudiante 8, E1) y "trabajar en grupo" (Estudiante 9, E1), queriendo señalar la importancia de "trabajar unidos" (Estudiante 19, E1). Destacan de este trabajo colectivo que "lo importante es apoyarse entre sí, para poder lograr sus metas. Especialmente un mundo más verde y limpio" (Estudiante 17, E1). De esta manera concuerdan que para lograr los objetivos propuestos necesitamos de una acción coordinada y grupal.

Se trata de una colectividad "comprometida en ayudar" (Estudiante 7, E5) y con “disposición para trabajar" (Estudiante 17, E5). En los establecimientos educacionales "se 
necesita todo el apoyo posible; alumnos, apoderados, profesores, etc. Lo más importante es que todas las personas estén dispuestas a cambiar el mundo que tenemos, transformándolo en uno mejor" (Estudiante 3, E5). Para lograr esa disposición y compromiso agregan que es necesario también "pedir la opinión de tus amigos" (Estudiante 6, E1) y de "los demás" (Estudiante 9, E1).

Algunas/os van más allá respecto de la participación y señalan que es esencial "pedir apoyo a los niños, pedirle una opinión a las niñas de su establecimiento" (Estudiante 15, E1), por esto "debes pedirle la opinión a los niños para saber qué es lo que opinan ellas de su establecimiento y para ver que le hace falta" (Estudiante 16, E1). En ese sentido, se debe hablar del tema tanto "con niños y niñas" como con "adultos" (Estudiante 18, E6).

También destacan la importancia de realizar acciones comunales y escolares para conmemorar una temática, o para visibilizar las acciones que realizan durante el año los establecimientos educacionales y sus participantes. Por este motivo, algunos celebran "el día mundial del medio ambiente" (Estudiante 20, E2) o "el día del agua" (Estudiante 19, E3).

\subsubsection{Acciones de (auto)formativas}

Muchas acciones escritas están relacionadas con la educación y la formación ambiental, en común podríamos decir que estas acciones tienen que "tener una orientación ecológica" (Estudiante 7, E5). A su vez, señalan respecto de la metodología, que es necesario "enseñar formas divertidas de mejorar el mundo" (Estudiante 1, E5), como por ejemplo "hacerles charlas recreativas sobre el cuidado del medio ambiente, juegos recreativos, reciclado de papel y mostrándoles vídeos a los niños de cómo reciclar" (Estudiante 14, E5). Otra/o estudiante piensa que "puedes enseñarles con juegos y dinámicas a reciclar y cuidar la naturaleza [...]. Hacer un taller entretenido y enseñar todo sobre el medio ambiente" (Estudiante 18, E6). En este mismo sentido, un/a estudiante recomienda "dar una buena educación ecológica a los estudiantes, acostumbrarnos a reciclar y recoger basura y sobre todo dedicar mucho tiempo" (Estudiante 12, E5).

La idea de "tener un taller de medio ambiente" (Estudiante 26, E6) es reiterada por muchos de los y las escolares en sus cartas. Señalan que en estos talleres se puede "hablar de reciclaje, [...] explicarle a todos donde deben botar la basura y explicarle que el planeta es importante" (Estudiante 16, E6), que son también para que las personas puedan por tanto aprender "a reciclar y a cuidar el planeta, que es muy importante para los seres vivos" (Estudiante 6, E2). Las acciones educativas, como talleres y charlas, contribuyen entonces a que las personas comprendan la importancia de lo ambiental y que aprendan a cómo actuar en pos del medioambiente.

Algunos/as creen que para lograr que estas acciones educativas sean de calidad es necesario "tener una profesora tan buena como la tía K" (Estudiante 3, E1) o que, para ellos, en el desarrollo de lo ambiental en su escuela, "lo más importante es la profesora N" (Estudiante 20, E5), reconociendo la importancia de los/as docentes. Por último, como acciones educativas que reflejan la profundidad del trabajo medioambiental en la escuela, mencionan la importancia del programa del Ministerio del Medioambiente llamado "los Forjadores Ambientales" (Estudiante 24, E5) y que, como consecuencia de todas estas iniciativas de educación, los establecimientos educacionales pueden "obtener un sello medio ambiental" (Estudiante 12, E5). En la Tabla 2 se esboza una síntesis de todas las acciones recién expuestas. 
Estudios Pedagógicos XLVII N 1: 281-302, 2021

SALVEN NUESTOR PALENTA: ANÁLISIS PROSPECTIVO DE 150 NIÑOS, NIÑAS Y ADOLESCENTES DE CHILE SOBRE LA EDUCACIÓN AMBIENTAL DEL FUTURO

Tabla 2. Acciones proambientales identificadas por niños, niñas y adolescentes

\begin{tabular}{|c|c|c|}
\hline Tipo de acción & Ámbito de acción & Acción específica \\
\hline \multirow{3}{*}{$\begin{array}{l}\text { Gestión } \\
\text { sustentable }\end{array}$} & $\begin{array}{l}\text { Gestión de residuos } \\
\text { escolares y } \\
\text { comunitarios }\end{array}$ & $\begin{array}{l}\text { - Reutilizar desechos orgánicos (e.g. compostaje). } \\
\text { - Acciones "R" con residuos escolares y domiciliarios } \\
\text { sólidos. } \\
\text { - Instalar contenedores y sistema de recuperación } \\
\text { diferenciados. }\end{array}$ \\
\hline & Producción vegetal & $\begin{array}{l}\text { - Plantar árboles tanto en la escuela como en espacios } \\
\text { públicos. } \\
\text { - Colocar unidades de producción vegetal (e.g. } \\
\text { jardineras, enredaderas o musgos en murallas de la } \\
\text { escuela). } \\
\text { - Campañas de arborización nativa. }\end{array}$ \\
\hline & $\begin{array}{l}\text { Construcción de } \\
\text { infraestructura }\end{array}$ & $\begin{array}{l}\text { - Puntos limpios tanto intra como extra escolares. } \\
\text { - Construir una vermicompostera. } \\
\text { - Construir un vivero. } \\
\text { - Instalaciones de energías renovables (e.g. paneles } \\
\text { solares). } \\
\text { - Áreas verdes públicas. }\end{array}$ \\
\hline \multirow{4}{*}{$\begin{array}{l}\text { Cuidado y } \\
\text { preservación }\end{array}$} & $\begin{array}{l}\text { Cuidado de los seres } \\
\text { vivos }\end{array}$ & $\begin{array}{l}\text { - Acciones de conservación de la flora (e.g. riego). } \\
\text { - Campañas de prevención de incendios forestales. } \\
\text { - Tenencia responsable de mascotas. } \\
\text { - Responsabilidad social sobre el cuidado de los } \\
\text { animales en condición de calle. }\end{array}$ \\
\hline & $\begin{array}{l}\text { Cuidado de la salud de } \\
\text { las personas }\end{array}$ & $\begin{array}{l}\text { - Campañas de prevención de consumo de tabaco. } \\
\text { - Campañas de lugares sin humo. }\end{array}$ \\
\hline & $\begin{array}{l}\text { Cuidado de los } \\
\text { recursos energéticos }\end{array}$ & $\begin{array}{l}\text { - Acciones de cuidado de la luz (e.g. cambiar } \\
\text { ampolletas). } \\
\text { - Acciones de cuidado del agua (e.g. poner afiches } \\
\text { junto a la canilla). }\end{array}$ \\
\hline & $\begin{array}{l}\text { Limpieza y aseo } \\
\text { escolar }\end{array}$ & - Participación activa de limpieza de salas y patios. \\
\hline \multirow{4}{*}{$\begin{array}{l}\text { Comunicación } \\
\text { y organización }\end{array}$} & Difusión & $\begin{array}{l}\text { - Voz a voz desde estudiantes. } \\
\text { - Campañas informativas (e.g. redes sociales y radios } \\
\text { locales). }\end{array}$ \\
\hline & Trabajo en equipo & $\begin{array}{l}\text { - Colaboración y coordinación entre actores escolares. } \\
\text { - Compromiso de la comunidad educativa } \\
\text { (engagement). }\end{array}$ \\
\hline & $\begin{array}{l}\text { Participación infantil } \\
\text { y adolescente }\end{array}$ & $\begin{array}{l}\text { - Acciones promovidas desde los/as estudiantes. } \\
\text { - Acciones de consulta a estudiantes (e.g. decidir } \\
\text { sobre proyectos ambientales escolares). }\end{array}$ \\
\hline & Acciones comunales & $\begin{array}{l}\text { - Actos conmemorativas (e.g. día de la tierra, año } \\
\text { nuevo mapuche). } \\
\text { - Eventos para visibilizar las acciones escolares y de } \\
\text { otros actores sociales (e.g. carnaval del reciclaje). }\end{array}$ \\
\hline
\end{tabular}




\begin{tabular}{|c|c|c|}
\hline \multirow{3}{*}{$\begin{array}{l}\text { (Auto) } \\
\text { formativas }\end{array}$} & $\begin{array}{l}\text { Metodología } \\
\text { y didáctica }\end{array}$ & $\begin{array}{l}\text { - Educación lúdica y activa. } \\
\text { - Educación práctica y basada en problemas locales. }\end{array}$ \\
\hline & Instancias educativas & $\begin{array}{l}\text { - Taller ambiental permanente. } \\
\text { - Capacitaciones a la Comunidad educativa. } \\
\text { - Charlas expositivas desde/ y hacia la Comunidad } \\
\text { educativa. }\end{array}$ \\
\hline & $\begin{array}{l}\text { Ambientalización } \\
\text { institucional }\end{array}$ & $\begin{array}{l}\text { - Docentes exclusivamente dedicados a la Educación } \\
\text { Ambiental. } \\
\text { - Sellos ambientales e inclusión del medioambiente } \\
\text { en misión, visión y otros instrumentos institucionales. }\end{array}$ \\
\hline
\end{tabular}

Fuente: Elaboración propia.

\section{2. "LA ACCIÓN CLIMÁTICA DEPENDE DE TODOS": PROBLEMÁTICAS, LUGARES Y ENTES FUNDAMENTALES EN LA CONSTRUCCIÓN DEL FUTURO}

Tal como en el apartado anterior, aquí se da uso al eslogan de la COP 25 para señalar que, dado que la acción climática depende de todos, sería justo y necesario saber respecto de esos todos/as que hacemos referencia y sobre aquellas necesidades climáticas de las cuales depende aquel colectivo. Aquí se agruparon ciertas problemáticas locales, como actores/ actrices y lugares claves identificados en las cartas de los y las NNA y que, de cierta forma, claman por hacernos responsables.

\subsubsection{Problemáticas locales por resolver}

Una de las grandes problemáticas que los/as estudiantes consideran debemos afrontar, es la producción desenfrenada de residuos, por lo que invitan a "no botar la basura porque contamina el ambiente" (Estudiante 13, E4) y a que no se "usen más bolsas plásticas" (Estudiante 6, E3). Así, el problema de la basura fue el más aludido de todas las problemáticas locales. Entienden que se trata de una acción que debemos detener, sobre todo en lugares al aire libre y que es necesario que "cuando vayan de paseo al campo o al río se llevan su basura de vuelta" (Estudiante 18, E3). Esto lo señalan sobre todo estudiantes rurales porque "los adultos vienen de Los Ángeles a ensuciar el campo donde nosotros vivimos" (Estudiante 22, E3).

Misma conciencia respecto de lo perjudicial de los residuos en el agua, por lo que llaman a "no tirar basura a los lagos o mares para no contaminar el agua y para no enfermar a los animales y cuidar las plantas del mar" (Estudiante 19, E2), en específico a "no botar plástico en el mar" (Estudiante 10, E3) y en general a "no botar basura en el océano ni en ningún lugar del mundo" (Estudiante 5, E2). Por lo mismo piden "no botar petróleo en el mar" (Estudiante 7, E3).

Respecto de los seres vivos, los y las estudiantes rurales señalan que les gustaría "que no boten los animales en el camino y basura en el campo" (Estudiante 22, E3), así otros/ as piden que "no maten a los animales en peligro de extinción" (Estudiante 1, E3). Buscan que los animales no se vean afectados ni por maltrato directo ni por la contaminación de sus condiciones de vida. Otro ser vivo afectado son los árboles nativos, debido a la tala. 
Un/a estudiante al respecto señala que "no me gusta que corten los árboles nativos, porque así matamos a los árboles más importantes del país" (Estudiante 15, E3).

Otro problema es la fuerte contaminación atmosférica que genera en su comuna el sistema de calefacción a leña, por lo que piden "reducir el humo de las estufas" (Estudiante 4, E1), "no usar leña verde, por el humo" (Estudiante 19, E6) o "no hacer fuego regularmente porque contamina mucho" (Estudiante 18, E1). Según algunos/as, esto sería fundamental porque "el medio ambiente lo cuidan las plantas [...] y lo más importante el aire" (Estudiante 31, E5) por lo que es necesario "que reduzcan el humo del país para que se pueda respirar mejor y no haya tanta contaminación atmosférica" (Estudiante 2, E1).

Otra problemática mencionada son los incendios, experiencia que más de alguno/a ha vivido en la zona entre los años 2017-2019. Al respecto, señalan que es importante "no encender los árboles de los bosques" (Estudiante 11, E3) y que por seguridad es mejor "no hacer fuego cerca" (Estudiante 10, E3), "no fumar, porque puede aún estar prendido y eso puede provocar incendios" (Estudiante 13, E4) y derechamente "que no hagan muchas fogatas" (Estudiante 9, E6). A su vez, son conscientes de la importancia de "no quemar los bosques, porque se pueden morir los animales" (Estudiante 15, E4).

\subsubsection{Entes y lugares claves del presente-futuro}

Los y las estudiantes confieren importancia a cuatro entes de cara al futuro: las personas, las escuelas, las empresas y los países. De las primeras esperan un comportamiento proambiental y la capacidad de involucrarse en iniciativas como las que hemos mencionado en subcategorías anteriores. A los establecimientos educacionales los ven como actores donde suceden o pueden llegar a suceder una gran cantidad de acciones y procesos proambientales, inclusive en aquellos más desventajados, como lo ilustra este/a NNA:

Sí hay una escuela que tiene solo tierra, pueden cambiarla y agregarle cosas nuevas, como: pasto, flores canchas y si hay una parte con forma de cuadrado hay que poner un árbol que en primavera crezcan flores de color rosa (Estudiante 5, E5).

Respecto de las empresas, señalan que estas deben "ser buenas" (Estudiantes 5, E1), pues actualmente "los tubos de algunas empresas nos pueden enfermar" (Estudiante 13, E4). De manera similar, señalan que un desafío de las naciones es realizar acciones para "que haya menos contaminación en el mundo, país, o más lugares verdes para que las personas puedan jugar libres" (Estudiante 21, E6), por lo mismo dicen que es necesario "decirles a los demás que traten de cuidar su medioambiente o su país" (Estudiante 12, E1). Una manera de decirles según un/a escolar puede ser "hacer estos talleres en todo Chile para intentar impulsar a Chile a ser un país sin contaminación” (Estudiante 12, E2).

Hablan también de otros lugares de carácter local como las comunas, el campo, la ciudad, las calles, los parques, los jardines y los patios de las escuelas. Aun así, los lugares más mencionados son aquellos considerados "naturales": bosques, mares, lagos, ríos o canales. Dentro de estos, los acuáticos son los más indicados, reiterándose el llamado a "recoger la basura en los ríos, no botar el plástico en los mares, ríos y océanos" (Estudiante 11, E3) y a "limpiar tus ríos, lagos, para que saques agua limpia para regar la tierra y colocar más plantas" (Estudiante 3, E6), en definitiva, realizar acciones que "cuiden el agua de los ríos y canales. Cuidar el paisaje que es muy hermoso" (Estudiante 20, E3). 
Una de sus razones para tener gran consideración del agua, el mar y los océanos es que al "no tirar basura a los lagos o mares" se busca evitar "contaminar el agua, para no enfermar a los animales y cuidar las plantas del mar" (Estudiante 19, E2). Un/a escolar consciente de las consecuencias de las acciones humanas que "las personas que fuman se enferman y también los peces, los tiburones y los pulpos, o cualquier cosa del mar se intoxica, porque cuando tu tiras el cigarro al mar están los peces, así que no se fuma, acuérdense que no hay que fumar y el que no sabe, que lea este mensaje" (Estudiante 18, E4). En la Tabla 3 se observa una síntesis de esta categoría diferenciada según el eje abordado por los/as NNA.

Tabla 3. Problemáticas, lugares y entes críticos identificados por niños, niñas y adolescentes

\begin{tabular}{|l|l|}
\hline \multicolumn{1}{|c|}{ Eje temático } & \multicolumn{1}{c|}{ Particularidad identificada } \\
\hline $\begin{array}{l}\text { Problemáticas } \\
\text { socioambientales locales }\end{array}$ & $\begin{array}{l}\text { Mala gestión de los residuos (basura). } \\
\text { Contaminación de las aguas. } \\
\text { Tala de árboles (nativos). } \\
\text { Maltrato animal (especies en extinción). } \\
\text { Contaminación atmosférica (leña intradomiciliaria). } \\
\text { Incendios forestales. }\end{array}$ \\
\hline Entes y actores claves & $\begin{array}{l}\text { Personas (conciencia ambiental). } \\
\text { Instituciones educativas (ambientalización institucional). } \\
\text { Empresas (responsabilidad ambiental empresarial). } \\
\text { Países (responsabilidad social y gubernamental). }\end{array}$ \\
\hline $\begin{array}{l}\text { Lugares y espacios } \\
\text { críticos }\end{array}$ & $\begin{array}{l}\text { Espacios locales (e.g. barrios, campo, ciudad, calles, parques y patios } \\
\text { escolares). } \\
\text { Espacios globales (e.g. bosques, océanos, mares, lagos o ríos). }\end{array}$ \\
\hline
\end{tabular}

Fuente: Elaboración propia.

\section{DISCUSIÓN Y CONCLUSIONES}

Ir al futuro es como emprender la odisea del espacio antes de que despunte en el horizonte la luz de una estrella aún no nacida que nos marcara el rumbo en el oscuro firmamento; como salir a circunnavegar el planeta sin saber que la Tierra es redonda y que detrás nos esperan las Indias; es ir con la pulsión de navegar, porque nos es más preciso que la vida, sin entender lo por-venir que nos espera, sin saber qué es ese más allá de la realidad. Así, el pensamiento vislumbra en la penumbra un futuro virtual pero posible, más allá del umbral de lo conocido, de la realidad fijada y deslumbrada por el iluminismo de la razón (Leff, 2011, p. 3).

Como bien lo ilustra Leff, preguntarse con los/as NNA sobre el futuro, supone anteponerse a lo aún no vivido en pos de delinear un rumbo en lo oscuro. Es abrir espacios para la vital expresión de esa pulsión de navegar, en pos de vislumbrar ese futuro virtual 
pero posible. Alinearse con la construcción de un saber ambiental otro que va sabiendo y conociendo, mediante va reconstruyendo el "proceso de creación de lo posible, un poderser que pasa por lo pensable" y lo decible, pero también por la asimilación de aquello pensado con lo posible, y con "su viabilidad a través de la realidad social existente" (Leff, 2011, p. 4).

Se trata, en palabras de Tréllez (2002), de configurar un futuro futurable (posible y deseable) que represente los intereses, ideas, perspectivas y propuestas de las múltiples comunidades que conforman y dinamizan niños, niñas, adolescentes y jóvenes. Permitir mediante ejercicios educativos, cívicos y proambientales, el encontrar un espacio sobre el cual labrar rutas de consenso en torno al mañana que les gustaría vivir a estos/as personas y colectividades. Cumplir con la promesa de devolverles recursivamente y de facto el futuro.

En esta línea, como en ejercicios anteriores (Hicks, 2014; Ojala, 2015), este estudio permite evidenciar que las experiencias de EA contribuyen a que los NNA se formulen una imagen positiva y constructiva sobre el futuro, en la cual pueden ser agentes activos. Incluso, que producto de este tipo de acciones son capaces de elaborar con claridad y pertinencia una serie de medidas que permitirían a futuro mejorar nuestras condiciones de existencia (Malone, 2013; Tsevreni \& Panayotatos, 2011) y nuestro campo de acción y estudio.

La presente investigación permite constatar un grado de profundidad de análisis relacionable al alcanzado por ejercicios anteriores. En primer lugar, una serie de estudios han demostrado que a partir de la EA los NNA pueden formular imágenes claras y críticas sobre el futuro global, a partir de las cuales se sienten incentivados a actuar en pos del medioambiente (Ojala, 2015); en segundo lugar, otras investigaciones han evidenciado que los NNA pueden condensar sus visiones de futuro en medidas concretas relacionadas con sus espacios locales como lo son barrios y escuelas (Malone, 2013; Tsevreni \& Panayotatos, 2011); en tercero, esta investigación permite afirmar que estas visiones y medidas del futuro pueden ser condensadas en un pool de acciones, provenientes de sus experiencias y de su capacidad de imaginar, y que beneficiarían tanto a la EA local, a la realizada en otras latitudes, como a la sociedad en general.

Aún más, estas estrategias sugeridas por los NNA son igualmente validas que las provenientes del mundo adulto, dado que coinciden con aquellas acciones educativas que obtienen mejores resultados en la $\mathrm{EA} / \mathrm{EpCC}$, según revisiones recientes (Monroe, Plate, Oxarart, Bowers, \& Chaves, 2019). Tal como en el citado estudio aquí se sugiere que la metodología educativa debe ser activa, didáctica y basada en problemas locales o significativos. Así mismo, se destaca la importancia de los intercambios de experiencias, de instalar proyectos tanto escolares como comunitarios, y de contribuir a corregir las concepciones y conductas erróneas o dañinas del medioambiente.

En este sentido, cabe considerar las propuestas y visiones de estos/as escolares como potenciales políticas, programas o acciones medioambientales que pueden realizarse tanto en el país, como en las diversas comunas y escuelas. Paralelamente a esto, muestran ideas relacionadas con problemáticas socioambientales tanto locales como globales. Esto habla de una capacidad de identificar problemas en los distintos niveles que se presentan, lo que según Gadotti (2008) puede significar una competencia importante para la construcción de una sociedad más sustentable y justa, en la medida que indicaría un conocimiento más profundo del propio territorio y de la crisis climática global que se nos presenta, una habilidad de análisis glocal. 
Los resultados del estudio coinciden con otros reportes nacionales, donde se remarca la importancia que le dan las/os escolares chilenos a acciones proambientales, tales como utilizar energía renovable, reciclar, cuidar los recursos o mantener los espacios limpios y agradables (Cortés, Cabana-Villca, Vega-Toro, Aguirre-Sarmiento \& Muñoz-Gómez, 2017; Gädicke-Robles, Ibarra-Palma \& Osses-Bustingorry, 2017). También existen coincidencias en cuanto a la importancia que le dan al trabajo colectivo de la comunidad educativa en el desarrollo de la EA, siendo vital el rol que desempeñan sus docentes (Cortés et al., 2017; Torres-Rivera, Benavides-Peña, Latoja-Vollouta \& Novoa-Contreras, 2017). Esto puede deberse a que, tanto las mencionadas acciones como el centrarse en la comunidad educativa, forman parte de los lineamientos de los programas públicos de EA en Chile (Ministerio de Medio Ambiente, 2018).

A su vez, presenta similitudes con otros estudios tanto nacionales como internacionales, donde los/as NNA han podido identificar una serie de problemáticas socioambientales que afectan a sus comunidades y que requieren de una solución prioritaria (Gädicke $e t$ al., 2017; García-Pérez \& Moreno-Fernández, 2015). En suma, esta investigación, como otras, indica que los y las niñas, niños, adolescentes y jóvenes son plenamente capaces de imaginar, construir y señalar el futuro ambiental que desean, como también de actuar críticamente frente el presente, siendo probablemente los/as adultos/as quienes ponen las mayores barreras y dificultades para tales ejercicios (Mackey, 2012).

Esto es aún más relevante si consideramos que los presentes resultados permiten formarse un imaginario distinto al catastrófico, un escenario en el cual, producto de la correcta acción humana, es posible disfrutar del medio ambiente sin sacrificarlo, sin poner en riesgo nuestra especie y "nuestor palenta" (Estudiante 25, E3). Así visto, salvar nuestor palenta no es utopía, si no posibilidad de existencia y de acción colectiva.

$\mathrm{Al}$ decir de Leff (2011), los resultados muestran imágenes y futuros en disputa en el marco de racionalidades que podríamos llamar "sustentables", pues NNA establecen una relación de sentido entre educación e infraestructura; cuidado y juego, contaminación y maltrato, ritos medioambientales y pueblos indígenas, futuro y acción (Hicks, 2014; Malone, 2013; Ojala, 2015). Así, los NNA nos invocan a creer que tal catástrofe es eludible mediante las acciones de gestión sustentable, de cuidado del medioambiente, de organización social y comunicación y de (auto)educación, si es que se tiene en consideración también, ciertos entes, lugares y problemáticas $(\mathrm{g})$ locales.

$\mathrm{Al}$ respecto de estos resultados, una limitación de este estudio es no haber realizado estas categorizaciones con los NNA partícipes. En pos de lograr una mayor protagonismo de los/as escolares, futuras investigaciones podrían discutir estos resultados con ellos/ as, con el fin de volverlos más precisos o de presentarlos como un petitorio o propuesta concreta a sus autoridades locales. Este reto supone dar plena voz, veto y voto a sus opiniones y propuestas, incluirlas en planificaciones locales, municipales e inclusive nacionales, puesto que su retroalimentación de primera línea nos acerca a sus territorios y nos permite mejorar las intervenciones socioambientales, su receptividad, efectividad y sustentabilidad.

En esta misma línea, sería interesante ver los diferentes ecos que generan estas medidas en académicos o expertos en EA, como también en gestores estatales, municipales y comunitarios del tema. Un primer paso en esa vía es diseminar las acciones y nodos identificados, después incentivar ejercicios de priorización colectiva que incluya a diversos grupos adultos como los antes mencionados, pero por sobre todo a NNA de 
Estudios Pedagógicos XLVII N ${ }^{\circ}$ : 281-302, 2021

SALVEN NUESTOR PALENTA: ANÁLISIS PROSPECTIVO DE 150 NIÑOS, NIÑAS Y ADOLESCENTES DE CHILE SOBRE LA EDUCACIÓN AMBIENTAL DEL FUTURO

diferentes partes y realidades del país. Una vez hecho esto, corresponde buscar los actores, liderazgos y financiamientos que soporten las diversas intervenciones aquí sugeridas.

A nivel investigativo, atendiendo diferencias sugeridas por estudios anteriores (Hicks, 1996; Ojala, 2015), sería interesante que futuros trabajos tomaran en especial consideración variables reportadas por este estudio pero no analizadas en profundidad como lo son el género de los/as participantes, la ruralidad que habitan, su grado de conocimiento y comportamiento ambiental, entre otros. Se puede hipotetizar que estas pueden tener cierto grado de incidencia en la imagen del futuro que tengan los NNA y en el tipo de acciones que sugieran para prefigurar este.

Para indagar más en ello, próximos estudios podrían ofrecer otras metodologías colectivas, corporales y centradas en el diálogo, para abordar este futuro futurable que proyectan las comunidades infanto-adolescentes. También sería interesante ver como otras metodologías propias de los Future Studies pueden ser aplicadas para conseguir tales objetivos. En suma, seguir ofreciendo más y mejores espacios, donde estos y estas, puedan sostener una visión de futuro en la cual se ven siendo participes de la configuración de una realidad social diferente, dinámica, sustentable, equitativa, apreciativa de lo diverso y lo múltiple, pacífica, justa y amorosa, en definitiva, otra.

\section{REFERENCIAS BIBLIOGRÁFICAS}

Alonso, A. (2018). Dirección de educación entrega PADEM a Concejo municipal. Noticias Municipalidad de Los Ángeles. Recuperado de: http://www.losangeles.cl/w/?p=47805 $(28 / 12 / 2018)$.

Cincera, J., Boeve-de Pauw, J., Goldman, D. \& Simonova, P. (2019). Emancipatory or Instrumental? Students' and Teachers' Perceptions of the Implementation of the Eco School Program. Environmental Education Research, 25(7), 1083-1104. doi:10.1080/13504622.2018.1506911.

Cortés, F., Cabana-Villca, R., Vega-Toro, D., Aguirre-Sarmiento, H. \& Muñoz-Gómez, R. (2017). Variables influyentes en la conducta ambiental en alumnos de unidades educativas, región de Coquimbo-Chile. Estudios Pedagógicos, 43(2), 26-46. doi:10.4067/S0718-07052017000200002

Fernández-Güell, J. M. (2011). Recuperación de los estudios del futuro a través de la prospectiva territorial. Ciudad y Territorio-Estudios Territoriales, (167), 11-32.

Gädicke-Robles, J., Ibarra-Palma, P., \& Osses-Bustingorry, S. (2017). Evaluación de las percepciones medioambientales en estudiantes de enseñanza media de la ciudad de Temuco, Región de La Araucanía. Estudios pedagógicos, 43(1), 107-121. doi:10.4067/S0718-07052017000100007

Gadotti, M. (2008). Education for Sustainability: A Critical Contribution to the Decade of Education for Sustainable Development. Green Theory and Praxis: The Journal of Ecopedagogy, 4(1), $15-64$.

Garcia-Pérez, I. \& Moreno-Fernández, O. (2015). El alumnado de primaria participante en el programa educativo Ecoescuelas ante las problemáticas socio-ambientales. De la perspectiva local a la glocal. Investigación en la Escuela, (87), 91-104.

González-Gaudiano, É. (2012). La representación social del cambio climático: una revisión internacional. Revista Mexicana de Investigación Educativa, 17(55), 1035-1062.

González-Gaudiano, E. \& Meira-Cartea, P. Á. (2020). Educación para el cambio climático:¿ educar sobre el clima o para el cambio? Perfiles Educativos, 42(168), 157-174. https://doi.org/10.22201/ iisue.24486167e.2020.168.59464

Hart, R. (2013). Children's participation: The theory and practice of involving young citizens in community development and environmental care. London: Routledge. 
Estudios Pedagógicos XLVII N ${ }^{\circ}$ 1: 281-302, 2021

SALVEN NUESTOR PALENTA: ANÁLISIS PROSPECTIVO DE 150 NIÑOS, NIÑAS Y ADOLESCENTES DE CHILE SOBRE LA EDUCACIÓN AMBIENTAL DEL FUTURO

Hicks, D. (1996). Preferred Futures 2020: Are Students Interested in Sustainable Futures? Australian Journal of Environmental Education, 12, 41-45. https://doi.org/10.1017/S081406260000416X (2006). Envisioning the future: The challenge for environmental educators. Environmental Education Research, 2(1), 101-108. https://doi.org/10.1080/1350462960020109 . (2014). Educating for hope in trubbeled times. Climate change and the transition to a postcarbon future. London, England: Trentham Books.

Intergovernmental Panel on Climate Change. (2018). Summary for Policymakers. In: Global Warming of $1.5^{\circ} \mathrm{C}$. Ginevra: IPCC.

Íñiguez-Rueda, L. \& Antaki, C. (1994). El análisis del discurso en psicología social. Boletín de psicología, 44(63), 57-75.

Kerret, D., Orkibi, H. \& Ronen, T. (2014). Green perspective for a hopeful future: Explaining green schools' contribution to environmental subjective well-being. Review of General Psychology, 18(2), 82-88. DOI: https://doi.org/10.1037\%2Fgpr0000006

Leff, E. (2011). La esperanza de un futuro sustentable: utopía de la educación ambiental. Revista Transatlántica de educación, 9, 93-103.

Liebel, M. \& Gaitán, L. (2019). El poder de los niños y niñas. Anotaciones sobre el protagonismo de movimientos infantiles en la actualidad. Sociedad e Infancias, 3, 15-20. https://doi.org/10.5209/ soci. 65352

Mackey, G. (2012). To know, to decide, to act: the young child's right to participate in action for the environment. Environmental Education Research, 18(4), 473-484. doi: https://doi.org/10.1080/ 13504622.2011 .634494

Malone, K. (2013). "The future lies in our hands": children as researchers and environmental change agents in designing a child-friendly neighbourhood. Local environment, 18(3), 372-395. DOI: $10.1080 / 13549839.2012 .719020$

Matos, Y., Pasek, E., Peña, M. L. \& Briceño, M. V. (2018). Participación Ciudadana para una Educación Ambiental Sustentable. Revista Scientific, 3(9), 233-255. DOI: https://doi. org/10.29394/Scientific.issn.2542-2987.2018.3.9.12.233-255

Ministerio del Medio Ambiente. (2018). Educación ambiental: Una mirada desde la institucionalidad ambiental chilena. Santiago de Chile: Ministerio del Medio Ambiente, División de Educación Ambiental y Participación Ciudadana.

Monroe, M. C., Plate, R. R., Oxarart, A., Bowers, A. \& Chaves, W. A. (2019). Identifying effective climate change education strategies: a systematic review of the research. Environmental Education Research, 25(6), 791-812.

Navas, M., Victoria, M., Martínez, M. \& Valdebenito, X. (2018). Marcos éticos para la investigación en educación con población infantil y juvenil: Hacia una propuesta de orientaciones. Documento de trabajo $\mathrm{N}^{\circ}$ 14. Santiago: Centro de Estudios MINEDUC.

O'Flaherty, J. \& Liddy, M. (2017). The impact of development education and education for sustainable development interventions: a synthesis of the research. Environmental Education Research, 7, 1031-1049. doi: https://doi.org/10.1080/13504622.2017.1392484

Ojala, M. (2012a). Hope and climate change: The importance of hope for environmental engagement among young people. Environmental Education Research, 18, 625-642. doi:10.1080/1350462 2.2011 .637157

. (2012b). Regulating worry, promoting hope: How do children, adolescents, and young adults cope with climate change? International Journal of Environmental and Science Education, 7(4), $537-561$.

. (2015). Hope in the Face of Climate Change: Associations With Environmental Engagement and Student Perceptions of Teachers' Emotion Communication Style and Future Orientation. The Journal of Environmental Education, 46(3), 133-148. DOI:10.1080/00958964.2015.1021662

Organización de Naciones Unidas. (2019). Informe de los objetivos de Desarrollo sostenible. New York: Naciones Unidas. ISSN: 2521-6899. 
Pramling-Samuelsson, I. (2011). Why we should begin early with ESD: The role of early childhood education. International Journal of Early Childhood, 43(2), 103. doi: https://doi.org/10.1007/ s13158-011-0034-x

Rodríguez, C. M. (2014). Pensamiento prospectivo: visión sistémica de la construcción del futuro. Análisis, 46(84), 89-104. doi: https://doi.org/10.15332/s0120-8454.2014.0084.05

Sauvé, L. (2013). Educación ambiental y ecociudadania. Dimensiones claves de un proyecto políticopedagógico. Revista Científica, (18), 12-23.

Schröder, L. M. U., Wals, A. E. \& van Koppen, C. S. A. (2020). Analysing the state of student participation in two Eco-Schools using Engeström's Second Generation Activity Systems Model. Environmental Education Research, 26(8), 1088-1111. DOI: 10.1080/13504622.2020.1779186.

Seamon, D. \& Gill, H. K. (2016). Qualitative approaches to Environment-Behavior Research: Understanding Environmental and Place Experiences, Meanings, and Actions. En R. Gifford (Ed.), Research methods for environmental psychology (pp. 115-136). UK: John Wiley \& Sons, Ltd.

Strife, S. (2012). Children's Environmental Concerns: Expressing Ecophobia. The Journal of Environmental Education, 43(1), 37-54. DOI: 10.1080/00958964.2011.602131

Torres-Rivera, L., Benavides-Peña, J., Latoja-Vollouta, C. \& Novoa-Contreras, E. (2017). Presencia de una Educación Ambiental basada en conocimiento, actitudes y prácticas en la enseñanza de las ciencias naturales en establecimientos municipales de la ciudad de Los Ángeles, Chile. Estudios Pedagógicos, 43(3), 311-323. doi:10.4067/S0718-07052017000300018

Tréllez, E. (2002). La educación ambiental comunitaria y la retrospectiva: una alianza de futuro. Tópicos en educación ambiental, 4(10), 7-21.

Tréllez, E. (2015). Educación ambiental comunitaria, participación y planificación prospectiva. Voces en el Fenix, (48), 185-191.

Trilla, J. \& Novella, A. M. (2001). Educación y participación social de la infancia. Revista Iberoamericana de Educación (OEI), 26, 137-164.

Tsevreni, I. \& Panayotatos, E. (2011). Participatory creation of a place-based teaching and learning methodology for children's participation and citizenship in the urban environment. Children Youth and Environments, 21(1), 293-309. DOI: 10.7721/chilyoutenvi.21.1.0293

World Economic Forum. (2019). The Global Risks Report 2019 (14 ed.). Suiza: World Economic Forum. Recuperado de: https://www.weforum.org/reports/the-global-risks-report-2019 (25/01/2019). (texto 12 , a la izquierda, normal) 